\title{
Evaluation of Different Seeding Methods for Cell-Seeded Collagen Matrix-Supported Autologous Chondrocyte Transplantation
}

\author{
Marco Viganò ${ }^{1}$ Gaia Lugano ${ }^{1}$ Alessandra Colombini ${ }^{1}$ Paola De Luca ${ }^{1}$ \\ Carlotta Perucca Orfei ${ }^{1}$ Enrico Ragni ${ }^{1}$ Laura de Girolamo ${ }^{1}$ \\ ${ }^{1}$ Orthopaedic Biotechnology Lab, IRCCS Istituto Ortopedico Galeazzi, \\ Milan, Italy \\ Joints 2018;6:215-219. \\ Address for correspondence Marco Viganò, PhD, Orthopaedic \\ Biotechnology Lab, IRCCS Istituto Ortopedico Galeazzi, Via Riccardo \\ Galeazzi, 4-20161 Milan, Italy \\ (e-mail: marco.vigano@grupposandonato.it).
}

\begin{abstract}
Keywords

- ACT-CS

- matrix

- chondrocytes

- cartilage

- intraoperative application

Purpose The aim of the present study was to evaluate different methods for the intraoperative seeding of chondrocytes on commercially available collagen I/III matrix, in the context of cell-seeded collagen matrix-supported autologous chondrocyte transplantation (ACT-CS).

Methods Human chondrocytes were enzymatically isolated from cartilage portion of discarded femoral heads of patients who underwent total hip replacement. Chondrocytes were cultured until passage 3 , and then used for the experiments. The cells $\left(5.0 \times 10^{5}\right)$ were suspended in two different volumes, 75 and $250 \mu \mathrm{L}$, and seeded on a matrix sample with a surface of $1 \mathrm{~cm}^{2}$ by means of a micropipette. Moreover, the direct immersion of the matrix in the cell suspension was evaluated as a possible protocol for chondrocyte seeding. Cell adhesion was allowed for 10, 30, or 60 minutes in all samples before evaluation.

Results Data showed that the seeding time did not affect cell viability and distribution, but there was a great difference between the two volumes of injection. In fact, the use of $75 \mu \mathrm{L}$ significantly reduced cell viability with respect to both $250 \mu \mathrm{L}$ seeding volume and the immersion protocol. Indeed, cell distribution resulted homogeneous in the samples seeded with the larger volume and with the immersion protocol.

Conclusion The use of $250 \mu \mathrm{L} / \mathrm{cm}^{2}$ volume or the immersion protocol for 10 minutes are valuable methods for chondrocyte seeding on collagen matrix in an intraoperative scenario. Clinical Relevance The protocol of chondrocyte seeding in ACT-CS is extremely variable among available literature reports. Chondrocytes adhesion to the matrix represents a crucial step in this methodology, and the present study provides in vitro indication for the choice of the seeding protocol in the context of ACT-CS.
\end{abstract}

\section{Introduction}

Cartilage is a nonvascularized connective tissue made of chondrocytes immersed in a dense extracellular matrix, mainly composed of collagen fibers and proteoglycans. ${ }^{1}$ Traumatic or degenerative events could lead to chondral and osteochondral lesions that may affect cartilage and the subchondral bone. ${ }^{2,3}$ The low grade of vascularization and the poor migration ability of chondrocytes within the tissue represent the two major limitations to cartilage self-repair, leading to healing failure and thus to pain, loss of function, and long-term complications. ${ }^{3-5}$ Hence, it is important to rely on a valid therapeutic approach to enhance tissue repair and avoid fibrotic scar formation. For this purpose, different received

December 22, 2017 accepted after revision June 10, 2019

published online

July 25, 2019
DOI https://doi.org/

10.1055/s-0039-1693458. ISSN 2282-4324.
Copyright (c) 2018 Georg Thieme Verlag License terms KG Stuttgart · New York
(요 (1) $\Theta$ 
surgical strategies have been proposed to restore or preserve hyaline cartilage, even though a successful treatment of these lesions remains challenging. ${ }^{6}$

The treatment of cartilage lesions comprises traditional operative approaches, such as joint debridement, ${ }^{7}$ drilling, and microfractures of the subchondral bone. ${ }^{8}$ However, these approaches are feasible in small chondral lesions only, while different techniques should be applied to treat larger defects. Indeed, for these kinds of lesions, a regenerative approach is preferred. For instance, matrix-assisted microfracture techniques like autologous matrix-induced chondrogenesis or autologous chondrocytes implantation (ACI), either associated with a collagen matrix, seem to provide more satisfactory and durable results in the treatment of medium-large chondral defects. $^{9-12}$ In this context, matrices are used with different applications and purposes. Indeed, in matrix-induced $\mathrm{ACI}$ chondrocytes may be injected in the lesion before suturing or directly cultured on the matrix for weeks before implantation, while in cell-seeded collagen matrix-supported autologous chondrocyte transplantation (ACT-CS) the cells are seeded on the matrix right before implantation, intraoperatively. These techniques represent an evolution of $\mathrm{ACI}$ and require a two-step surgery, the first where a cartilage biopsy is collected for chondrocytes isolation and culture, followed by a second procedure for cell and matrix implantation in the recipient site. ${ }^{13,14}$

One of the limitations of the techniques combining cells and matrices is the low reproducibility of the methods, mainly due to the differences in chondrocytes viability, number of cells, and seeding protocols, sometimes leading to conflicting outcomes. ${ }^{10,15-17}$ Thus, in the context of ACT$\mathrm{CS}$, different methods were evaluated to define the most effective protocol for chondrocytes seeding on a commercially available collagen I/III matrix.

The main purpose of this study was to in vitro investigate the effect of different protocols for chondrocytes seeding on the matrix, to identify the most effective approach, and provide valuable indications for the standardization of the procedures. The hypothesis of the study was that different seeding methods would influence cell adhesion and distribution of chondrocytes on the matrix in the context of ACT-CS.

\section{Methods}

\section{Human Chondrocytes Isolation and Culture}

Autologous human chondrocytes were enzymatically isolated from discarded hip joint cartilage portions of patients who underwent total hip arthroplasty at the Galeazzi Orthopedic Institute, Milan, Italy. All patients gave written consent to the collection of waste biological material. Briefly, cartilage tissue was harvested from the femoral head, minced, and incubated overnight at $37^{\circ} \mathrm{C}$ with $0.15 \%$ Collagenase type II (Worthington Biochemical Corporation, New Jersey, United States) in Dulbecco's modified Eagle medium (SigmaAldrich, Missouri, United States). Then, cells were counted and seeded at a density of $5.0 \times 10^{3} \mathrm{cell} / \mathrm{cm}^{2}$ and cultured in a humidified atmosphere at $37^{\circ} \mathrm{C}$ and $5 \%$ of $\mathrm{CO}_{2}$. When at $80 \%$ confluence, cells were harvested by trypsin (Lifetechnologies, California, United States) treatment and then seeded for further expansion until passage 3, when the experiments were performed.

\section{Human Chondrocytes Seeding on Collagen Matrix}

The bilayer collagen matrix used is composed of biocompatible porcine collagen type I/III (Chondro-Gide, Geistlich, Switzerland). The device has a compact layer which has a smooth surface and prevents the diffusion of the injected cells; the other layer of the matrix consists of a porous surface with a more loosely arrangement of collagen fibers that favors cell invasion and attachment.

To evaluate the suitable volume for cell seeding on Chondro-Gide, a preliminary absorption test was performed on $1 \mathrm{~cm}^{2}$ of the dry matrix.

The following volumes of blue color pigment solution ( $3 \%$ in phosphate-buffered saline), were tested: 50, 75, 100, 150, and $250 \mu \mathrm{L}$.

Three different seeding methods were adopted: small volume $(75 \mu \mathrm{L})$ or large volume injection $(250 \mu \mathrm{L})$ on the porous side of the matrix with a micropipette and matrix immersion in the cell suspension. According to the literature, ${ }^{11,18}$ we decided to use $0.5 \times 10^{6}$ cells $/ \mathrm{cm}^{2}$ suspended in the two volumes of interest. The more concentrated cell suspension $\left(5 \times 10^{5}\right.$ cell/75 $\left.\mu \mathrm{L}\right)$ was used for matrix immersion. Three seeding times, 10, 30, and 60 minutes, were tested with the purpose to identify the best time allowing for a complete and homogeneous cellular adhesion on the matrix in view of intraoperative seeding technique as usually done by many surgeons. ${ }^{19}$ For each condition, a qualitative and quantitative analysis of adhered cells were made by 4',6diamidino-2-phenylindole (DAPI) (Sigma-Aldrich) fluorescent staining and MTT assay (Sigma-Aldrich), respectively. Not-seeded matrices were prepared as controls.

\section{Viability Assay}

Metabolic activity of human chondrocytes seeded onto Chondro-Gide was measured by the MTT assay. After cell seeding with different protocols, the matrices were transferred in a $0.5 \mathrm{mg} / \mathrm{mL}$ solution of 3-(4,5-dimethylthiazol-2yl)-2,5-diphenyltetrazoliumbromide (MTT, Sigma-Aldrich) and incubated for 4 hours at $37^{\circ} \mathrm{C}$ and $5 \% \mathrm{CO}^{2}$, to allow the formation of formazan crystals in the proximity of viable cells. Then, the medium was removed and substituted with the same volume of dimethyl sulfoxide (Sigma-Aldrich) to solubilize the formazan crystals. Finally, the absorbance of the resulting solution was read at $570 \mathrm{~nm}$ (Victor X3, Perkin Elmer microplate, Massachusetts, United States).

\section{Immunofluorescence Assay}

Matrix specimens were fixed in 10\% formalin for 24 hours. Then, they were dehydrated in an increasing alcohol scale before embedding in paraffin and cutting into $3.5 \mu \mathrm{m}$ longitudinal sections. The slides were rehydrated in a decreasing alcohol scale and then permeabilized with a $0.5 \%$ Triton X-100 (Sigma-Aldrich) solution at room temperature (RT) for 10 minutes. After washing passages, the slides were incubated for 10 minutes at RT in the dark with DAPI (Sigma-Aldrich) to stain cells nuclei, and mounted with an aqueous mounting 
agent (Enzo Life Sciences, New York, United States). Photomicrographs of the matrix were captured through an Olympus IX71 fluorescence microscope and an Olympus XC10 camera (Olympus Corp, Tokyo, Japan).

\section{Statistical Analysis}

The experiments were conducted in triplicates. Results were analyzed by GraphPad Prism 5.0 software (California, United States). Data are expressed as mean \pm standard deviation. One-way analysis of variance (ANOVA) with Bonferroni's post hoc test was used for the comparison of samples in the different groups. Two-way ANOVA test with Bonferroni's post hoc test was applied to test the influence of seeding method and incubation time in all samples. A $p$-value of $<0.05$ was considered statistically significant.

\section{Results}

The results of the absorption test showed that $1 \mathrm{~cm}^{2}$ of the matrix was completely wet already by the use of $75 \mu \mathrm{L}$; increasing the volume of the blue color pigment solution lead to a progressive swelling of the matrix size till it started to leak noticeably when a volume of $250 \mu \mathrm{L}$ was used. Consequently, it was decided to compare the same number of cells seeded in a small $(75 \mu \mathrm{L})$ and a large volume $(250 \mu \mathrm{L})$ in the following experiments ( $\mathbf{- F i g .} \mathbf{1}$ ).

\section{Cell Viability}

The results of cell viability assay revealed that the time of seeding did not significantly affect cell viability, although a slight increasing trend with time was observed for each protocol. Indeed, the analysis of the contribution given by seeding method and incubation time revealed that they accounted for 87.21 and $4.30 \%$ of the total variance, respectively. Despite both being statistically significant $(p<0.0001$ and $p<0.01$, respectively), the influence given by time resulted negligible. On the other hand, samples seeded with high volume $(250 \mu \mathrm{L})$ and by immersion demonstrated significantly higher values compared with the $75 \mu \mathrm{L}$ seeding protocol (-Fig. 2). An evaluation of gross appearance of the matrix showed that in this case, the cells were not able to colonize the whole matrix, but rather generated a spot of concentrated cells in the middle ( - Fig. 3 ), negatively affecting cell viability.

\section{Distribution of Human Chondrocytes on Collagen Matrix}

To evaluate cells distribution and density on the porous surface of Chondro-Gide, DAPI staining was performed on

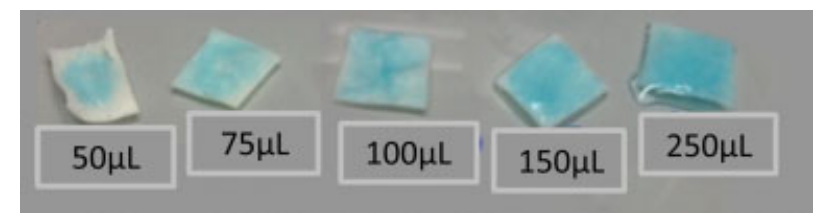

Fig. 1 Absorption test. Chondro-Gide matrices injected with different volumes of blue pigment solution.

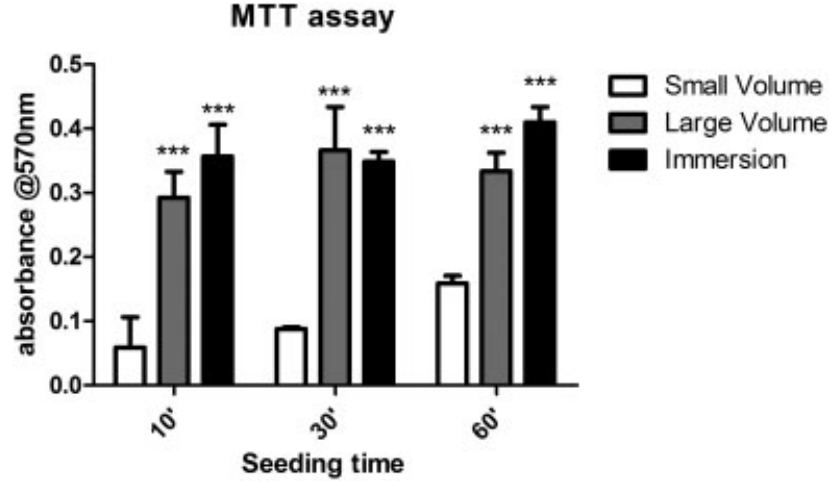

Fig. 2 Cell viability. The picture shows the results after 4 hours' incubation wit MTT assay of the seeded matrices. ${ }^{* * *} p<0.001$ vs. small volume.

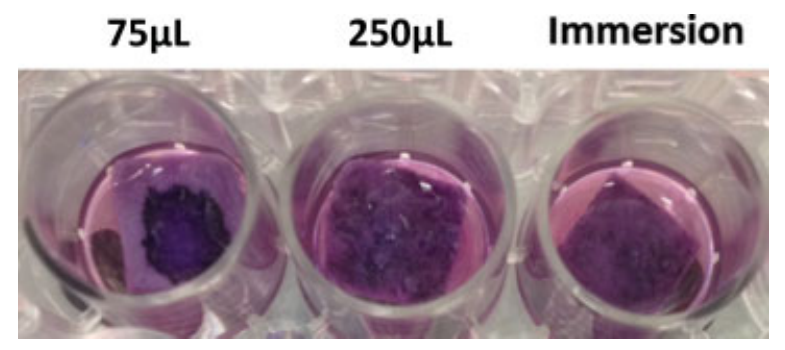

Fig. 3 Gross appearance of membranes after MTT assay.

paraffin-embedded samples. No differences among seeding time were observed. In particular, the results indicated that the immersion seeding protocol allowed for a uniform seeding on both sides of matrix, while the small and large volumes were injected on the porous surface of ChondroGide, thus resulting in a one-side seeding due to the bilayer structure of the matrix. Small volume injection appeared to increase the depth of seeding, and, indeed, it enhanced the concentration in the center of the matrix with respect to both large volume and immersion protocols. Conversely, the large volume allows for a more uniform and less concentrated seeding on the matrix (-Fig. 4).

\section{Discussion}

The present study aims to describe a reliable and reproducible method to seed chondrocytes on surgical devices in the contest of ACT-CS. Indeed, one of the limitation of this technique is represented by the different protocols adopted throughout the literature, limiting the comparison and the evaluation of surgical outcomes. ${ }^{5,19-21}$

Nowadays, the importance to improve the strategies of regenerative medicine to enhance cartilage healing is compelling. Among the different surgical and regenerative procedure proposed in the last decades, ACT-CS represents a valid strategy to overcome the limitations of traditional techniques ${ }^{11,13,19,20,22}$ and to partially restore the complex architecture of the hyaline cartilage, mitigating pain by reducing the friction surface phenomena. ${ }^{23}$ 
Blank

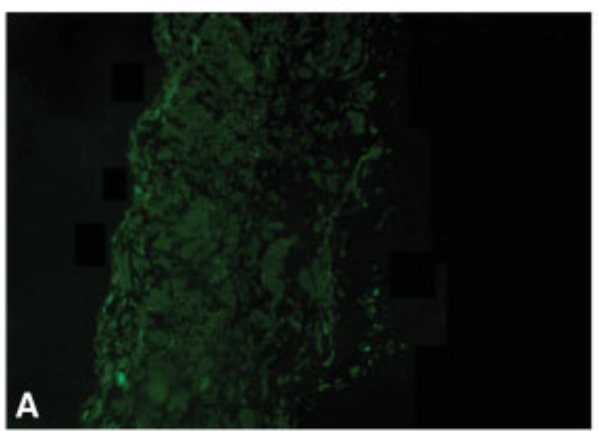

$250 \mu \mathrm{L}$

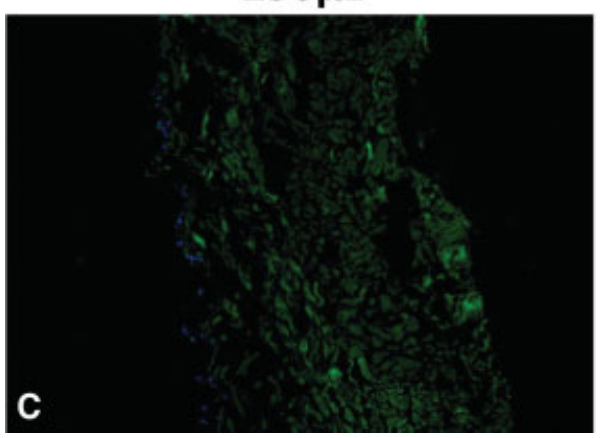

$75 \mu \mathrm{L}$

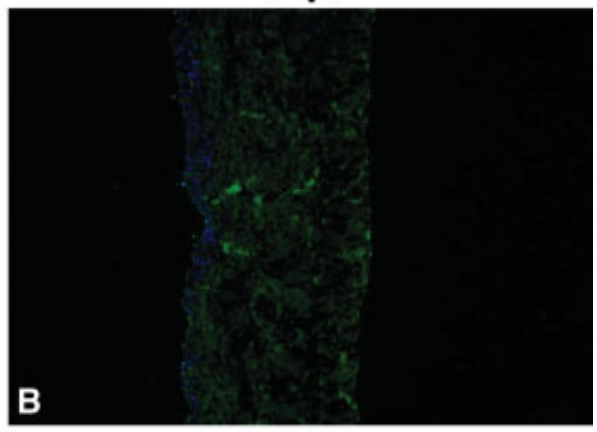

Immersion

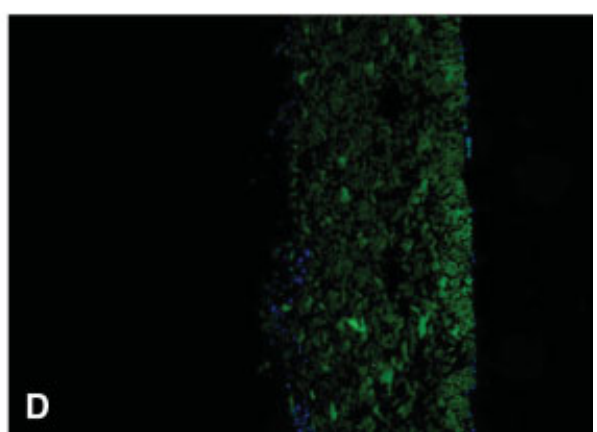

Fig. 4 4',6-Diamidino-2-phenylindole (DAPI) staining at 30 minutes. (A) A nonseeded membrane used as control. The small (B) and large (C) volumes (75 and $250 \mu \mathrm{L}$ ) allowed for cell distribution on the porous surface of the membrane, resulting in one-side seeding. (D) Immersion method determined a homogenous cell seeding on both sides of Chondro-Gide.

In our experiments, we used Chondro-Gide, a porcine collagen I/III matrix that represents a popular scaffold for this application. ${ }^{13,24-26}$ This device presents two different surfaces made of collagen type I/III, one porous intended to allow cell infiltration, and the other more compact, aimed to contain the cells within the lesion site, preventing their migration in the joint space. However, the compact side of the matrix also allows for cell adhesion as demonstrated by our results.

The optimal number of chondrocytes per square centimeter may be product-specific and thus our results might not be generalizable. According to the literature, ${ }^{11,18,27}$ in this study $5.0 \times 10^{5}$ cells $/ \mathrm{cm}^{2}$ were used, suspended in two different volumes of injection $(75$ and $250 \mu \mathrm{L}$ ). Our results confirmed that, with this method, chondrocytes were distributed on matrix surface, as previously reported by other authors. $^{28}$ Moreover, these findings showed that the small volume was not suitable to allow a uniform and effective cell adhesion on the matrix surface, while the use of the largest one increased the distribution of cells on the porous face of Chondro-Gide. Nevertheless, we applied the lower limit of the cell density reported in the literature, thus it is possible to hypothesize that increasing this parameter would enhance cell concentration and penetration within the matrix with the larger volume, similarly to what observed in the central portion of the matrix seeded with the smallest one. On the other hand, the immersion of the matrix in the cell suspension would represent a valid method for homogenous seed- ing, especially if the adhesion on both side of the matrix is desirable. Notably, negligible differences were observed among seeding times, suggesting that 10 minutes represents a sufficient time to allow for cell adhesion, both when cells were distributed by means of needle or immersion. This observation is particularly significant since the reduction of operative time can reduce costs and risks associated with surgeries.

Limitations of the present study are given by the use of laboratory equipment and techniques for cell seeding on the collagen matrix whose translatability in the context of a surgery room is yet to be confirmed. Moreover, the number of replicates was limited, even if the obtained results were statistically consistent.

In conclusion, in the context of ACT-CS, our results suggest using a volume of $250 \mu \mathrm{L}$ containing at least $5.0 \times 10^{5}$ cells per $\mathrm{cm}^{2}$ of matrix, for 10 minutes. Similarly, the immersion of the matrix in a cell suspension of $6.7 \times 10^{6}$ cells $/ \mathrm{mL}$ (corresponding to $5.0 \times 10^{5} / 75 \mu \mathrm{L}$ ) for the same seeding time would be a reliable method, but the adhesion of chondrocyte on both side of the matrix, and a lower level of penetration within the matrix, should be acknowledged.

Conflict of Interest

None declared. 


\section{References}

1 Sophia Fox AJ, Bedi A, Rodeo SA. The basic science of articular cartilage: structure, composition, and function. Sports Health 2009; 1(06):461-468

2 Aurich M, Hofmann GO, Rolauffs B, Gras F. Differences in injury pattern and prevalence of cartilage lesions in knee and ankle joints: a retrospective cohort study. Orthop Rev (Pavia) 2014;6 (04):5611

3 Madry H, Grün UW, Knutsen G. Cartilage repair and joint preservation : medical and surgical treatment options. Dtsch Arztebl Int 2011;108(40):669-677

4 Fahy N, Alini M, Stoddart MJ. Mechanical stimulation of mesenchymal stem cells: Implications for cartilage tissue engineering. J Orthop Res 2018;36(01):52-63

5 D’Anchise R, Manta N, Prospero E, Bevilacqua C, Gigante A. Autologous implantation of chondrocytes on a solid collagen scaffold: clinical and histological outcomes after two years of follow-up. J Orthop Traumatol 2005;6(01):36-43

6 Rosa D, Di Donato SL, Balato G, et al. How to manage a failed cartilage repair: a systematic literature review. Joints 2017;5(02): 93-106

7 McCormack R. The role of arthroscopy in the treatment of degenerative joint disease of the knee. BCMJ 2010;52(09): 439-441

8 Steadman JR, Rodkey WG, Briggs KK. Microfracture. Cartilage 2010;1(02):78-86

9 Minas T, Von Keudell A, Bryant T, Gomoll AH. The John Insall Award: a minimum 10-year outcome study of autologous chondrocyte implantation. Clin Orthop Relat Res 2014;472(01): 41-51

10 Saris D, Price A, Widuchowski W, et al; SUMMIT study group. Matrix-applied characterized autologous cultured chondrocytes versus microfracture: two-year follow-up of a prospective randomized trial. Am J Sports Med 2014;42(06):1384-1394

11 Steinwachs M, Peterson L, Bobic V, Verdonk P, Niemeyer P. Cellseeded collagen matrix-supported autologous chondrocyte transplantation (ACT-CS): a consensus statement on surgical technique. Cartilage 2012;3(01):5-12

12 Benthien JP, Behrens P. Autologous matrix-induced chondrogenesis (AMIC): combining microfracturing and a collagen I/III matrix for articular cartilage resurfacing. Cartilage 2010;1(01):65-68

13 Steinwachs M. New technique for cell-seeded collagen-matrixsupported autologous chondrocyte transplantation. Arthroscopy 2009;25(02):208-211

14 Marlovits S, Zeller P, Singer P, Resinger C, Vécsei V. Cartilage repair: generations of autologous chondrocyte transplantation. Eur J Radiol 2006;57(01):24-31

15 Bartlett W, Skinner JA, Gooding CR, et al. Autologous chondrocyte implantation versus matrix-induced autologous chondrocyte im- plantation for osteochondral defects of the knee: a prospective, randomised study. J Bone Joint Surg Br 2005;87(05):640-645

16 Crawford DC, DeBerardino TM, Williams RJ III. NeoCart, an autologous cartilage tissue implant, compared with microfracture for treatment of distal femoral cartilage lesions: an FDA phase-II prospective, randomized clinical trial after two years. J Bone Joint Surg Am 2012;94(11):979-989

17 Steinwachs M, Kreuz PC. Autologous chondrocyte implantation in chondral defects of the knee with a type I/III collagen membrane: a prospective study with a 3-year follow-up. Arthroscopy 2007;23 (04):381-387

18 Food and Drug Administration. Autologous Cultured Chondrocytes on a Porcine Collagen Membrane. Approval Letter; 2016 (BLA 125603/0). Available at: https://www.fda.gov/Biologics BloodVaccines/CellularGeneTherapyProducts/ApprovedProducts /ucm533177.htm. Accessed June 26, 2019

19 Gomoll AH, Ambra LF, Phan A, Mastrocola M, Shah N. Cell-seeded autologous chondrocyte implantation: a simplified implantation technique that maintains high clinical outcomes. Am J Sports Med 2017;45(05):1028-1036

20 Niemeyer P, Lenz P, Kreuz PC, et al. Chondrocyte-seeded type I/III collagen membrane for autologous chondrocyte transplantation: prospective 2-year results in patients with cartilage defects of the knee joint. Arthroscopy 2010;26(08):1074-1082

21 Behrens P, Bitter T, Kurz B, Russlies M. Matrix-associated autologous chondrocyte transplantation/implantation (MACT/MACI)5-year follow-up. Knee 2006;13(03):194-202

22 Kreulen C, Giza E, Walton J, Sullivan M. Seven-year follow-up of matrix-induced autologous implantation in talus articular defects. Foot Ankle Spec 2018;11(02):133-137

23 Makris EA, Gomoll AH, Malizos KN, Hu JC, Athanasiou KA. Repair and tissue engineering techniques for articular cartilage. Nat Rev Rheumatol 2015;11(01):21-34

24 Haddo O, Mahroof S, Higgs D, et al. The use of chondrogide membrane in autologous chondrocyte implantation. Knee 2004;11 (01):51-55

25 Cherubino P, Grassi FA, Bulgheroni P, Ronga M. Autologous chondrocyte implantation using a bilayer collagen membrane: a preliminary report. J Orthop Surg (Hong Kong) 2003;11(01):10-15

26 Schneider TE, Karaikudi S. Matrix-induced autologous chondrocyte implantation (MACI) grafting for osteochondral lesions of the talus. Foot Ankle Int 2009;30(09):810-814

27 Foldager CB, Gomoll AH, Lind M, Spector M. Cell seeding densities in autologous chondrocyte implantation techniques for cartilage repair. Cartilage 2012;3(02):108-117

28 Gigante A, Bevilacqua C, Ricevuto A, Mattioli-Belmonte M, Greco F. Membrane-seeded autologous chondrocytes: cell viability and characterization at surgery. Knee Surg Sports Traumatol Arthrosc 2007;15(01):88-92 